\title{
Acute Vertebral Artery Origin Occlusion Leading to Basilar Artery Thrombosis Successfully Treated by Angioplasty with Stenting and Thrombectomy
}

\author{
ARATA ABE ${ }^{1}$, SEIJI OKUBO ${ }^{1}$, SHIRO ONOZAWA ${ }^{2}$, MASATAKA NAKAJIMA ${ }^{1}$, \\ KENTARO SUZUKI ${ }^{1}$, MINA HARADA-ABE ${ }^{1}$, MASAYUKI UEDA ${ }^{1}$, \\ KEN-ICHIRO KATSURA ${ }^{1}$, YASUO KATAYAMA ${ }^{1}$ \\ ${ }^{1}$ Department of Internal Medicine, Division of Neurology, ${ }^{2}$ Department of Radiology, Nippon Medical School; Tokyo, Japan
}

Key words: stent, thrombosis, thrombectomy

\section{Summary}

Few reports have described the successful treatment of stroke caused by acute vertebral artery (VA) origin occlusion by endovascular surgery. We describe the case of a 68-year-old man who experienced stroke due to left acute VA origin occlusion. Cerebral angiography showed that the left VA was occluded at its origin, the right VA had hypoplastic and origin stenosis, and the basilar artery was occluded by a thrombus. The VA origin occlusion was initially passed through with a 0.035-inch guide wire. An angioplasty was performed, and a coronary stent was appropriately placed. The VA origin was successfully recanalized. A balloon-assisted guiding catheter was navigated through the stent and a thrombectomy was performed using the Penumbra system. The patient's symptoms gradually improved postoperatively. Balloon-assisted catheter guidance through a vertebral artery stent permitted a successful thrombectomy using the Penumbra system and may be useful for treating stroke due to VA origin occlusion.

\section{Introduction}

Proper positioning of a guiding catheter facilitates neuroendovascular interventions.

The present report describes a case in which a balloon-assisted technique was used to expe- dite the safe distal positioning of a flexible guiding catheter beyond a vertebral artery origin stent.

\section{Case Report}

A 68-year-old man with a history of hypertension, dyslipidemia, chronic renal failure, arteriosclerosis obliterans, and myocardial infarction, as well as a two-year history of angina pectoris and dialysis was investigated. The patient gave written informed consent for use of these data. The procedure was approved by the ethical review board of our department. An angiogram showed coronary triple-vessel disease and bilateral vertebral artery origin stenosis (Figure 1A,B). The right vertebral artery was hypoplastic, and circulation through the posterior communicating artery was confirmed. Anti-platelet drug administration was discontinued a week before a coronary artery bypass graft was to be performed. However, a day prior to surgery, the patient developed left-sided hemiparesis. Brain magnetic resonance imaging showed basilar artery (BA) occlusion and subacute left cerebellum infarction. Since the use of plasminogen activator was contraindicated, endovascular recanalization of the BA was planned. An angiogram revealed an occlusion at the left dominant vertebral artery origin (Figure 1C). Under local anesthesia, the lesion 

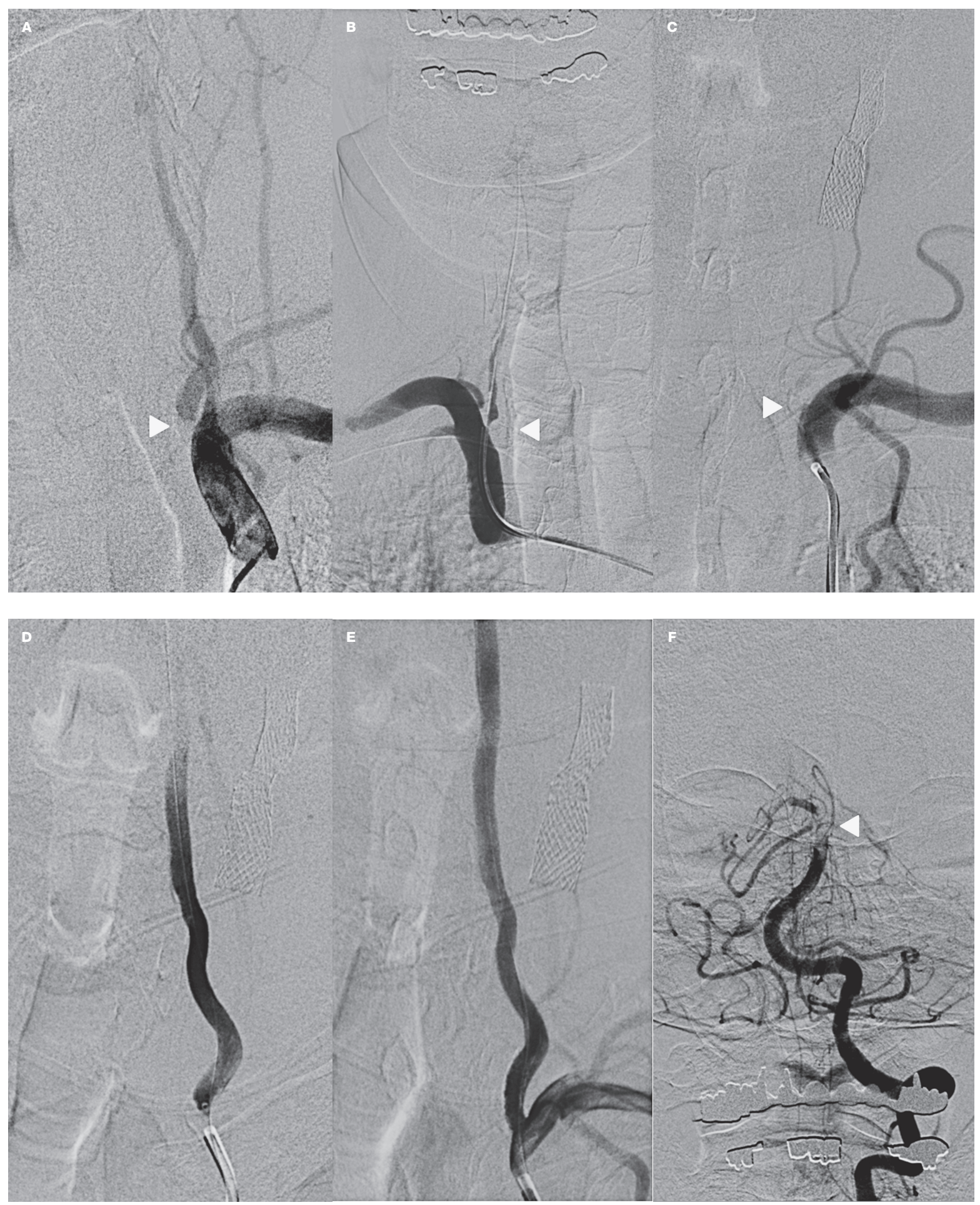

Figure 1 A) Angiogram demonstrating severe stenosis of the left vertebral artery origin (VA) (arrowhead). B) Angiogram demonstrating severe stenosis of the right VA origin (arrowhead). C) Angiogram demonstrating total occlusion of the left VA origin (arrowhead). D) Angiogram through the 0.035-inch guidewire confirming the patency of the lumen at the distal VA occlusion. E) Post stenting angiogram showing resolution of the occlusion and good antegrade perfusion. F) Angiogram demonstrating total occlusion of the top of the basilar artery (arrowhead). G) Resolution of the occlusion and good antegrade perfusion to the left posterior cerebral artery and right superior cerebellar artery, following thrombectomy performed using the Penumbra system (arrowhead). 
G

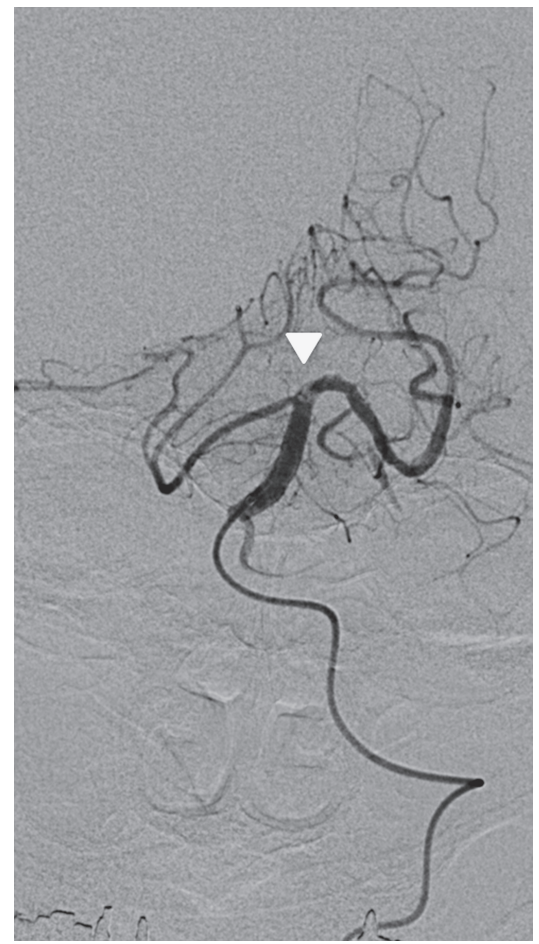

was traversed with a 0.035 -inch guide wire and the patency of the lumen distal to the lesion site was confirmed (Figure 1D). The guidewire was replaced with a 45-degree preshaped GT microwire (0.014-inch; Terumo, Tokyo, Japan). The diameter of the proximal segment of the left VA origin was approximately $3.8 \mathrm{~mm}$ and the lesion length, visually compared with a $6 \mathrm{Fr}$ guiding catheter (Cordis Endovascular, Miami Lakes, FL, USA), was approximately $10 \mathrm{~mm}$. A $4.0 \times 22 \mathrm{~mm}$ Integrity stent (Medtronic, Minneapolis, MN, USA) was advanced over the microwire and deployed at the lesion site. A subsequent angiogram resolved the occlusion at the top of the BA (Figure 1E). The balloon-expanded stent was reused. At a location adjacent to the stent, the balloon was inflated. The guiding catheter was then navigated to the proximal end of the balloon, which was then gradually deflated. The balloon was moved incrementally toward the distal position of the stent and was inflated. These steps were repeated, through which the guiding catheter was repositioned several times. Thus, the guiding catheter could be navigated beyond the vertebral artery origin stent without disturbing the placement of the vertebral artery origin stent.

The Penumbra system (Penumbra Inc,
Alameda, CA, USA) was then prepared. A 0.041-inch reperfusion catheter was positioned over a GT microwire inside the Tracker Excel-14 microcatheter (Target Therapeutics, Fremont, CA, USA), which was navigated coaxially through the 6 Fr guiding catheter to the P1 segment of the right posterior cerebral artery (PCA).

A gentle contrast injection procedure showed normal blood flow direction in the PCA and an extensive clot at the top of the BA. A separator was introduced in the reperfusion catheter, which was then connected to an aspiration pump, generating a vacuum of -20 in $\mathrm{Hg}$. A continuous aspiration-debulking process was facilitated by advancing and withdrawing the separator through the reperfusion catheter into the distal end of the clot and by withdrawing the tip of the reperfusion catheter stepwise. The process was repeated three times (Figure 1F).

Independent verification by a neurologist confirmed that the patient did not display any periprocedural neurological complications. At the one-month follow-up, slight left incomplete hemiparesis was apparent, and an antiplatelet treatment regimen was resumed to prevent thrombus recurrence.

\section{Discussion}

The current literature demonstrates the safety and efficacy of percutaneous transluminal angioplasty (PTA) with stenting for the treatment of VA origin stenosis and for endovascular recanalization of intracranial posterior circulation thrombosis concomitant with stenosis 1-4. Lin et al. ${ }^{3}$ and Noriaki et al. ${ }^{4}$ described seven cases of acute-subacute posterior circulation strokes due to VA origin occlusion/near occlusion or preocclusive stenosis associated with intracranial thrombosis. In all these cases, PTA with stenting and intracranial thrombolysis was performed. Five of the seven $(71 \%)$ patients had excellent immediate recovery, whereas two patients died, one of whom initially presented with coma. These results indicated that endovascular intervention might be a useful treatment option. In those studies, however, two patients presented with severely disabling stroke, which was ranked as grade 3 on the Modified Rankin Scale (mRS) and only three patients $(42 \%)$ had a good prognosis with a mRS score of $<2$. Angioplasty may be best reserved for cases in which thrombolysis alone fails. Angi- 
oplasty combined with thrombolysis may improve patient outcome. However, this procedure requires the safe passage of the guiding catheter through the stent. In the present case, correct positioning of the guiding catheter in the distal vertebral artery was facilitated by using a balloon stent to expand the vertebral artery origin stent. The successful outcome was probably due to the creation of less friction inside the stent by the formation of a smaller ledge between the balloon and the guiding catheter. However, there may still be a risk of the balloon bursting and an undesirable movement of the vertebral artery origin stent. These risks can be mitigated by effective communication between the two operators in charge, which can enable improved movement of the guiding catheter and an associated improvement in patient prognosis. Fields et al. ${ }^{5}$ reported two cases of Merci Retriever thrombectomy for the treatment of acute ischemic stroke due

\section{References}

1 Antoniou GA, Murray D, Georgiadis GS, et al. Percutaneous transluminal angioplasty and stenting in patients with proximal vertebral artery stenosis. J Vasc Surg. 2012; 55 (4): 1167-1177. doi: 10.1016/j. jvs.2011.09.084.

2 Stayman AN, Nogueira RG, Gupta R. A systematic review of stenting and angioplasty of symptomatic extracranial vertebral artery stenosis. Stroke. 2011; 42 (8): 2212-2216. doi: 10.1161/STROKEAHA.110.611459.

3 Lin DDM, Gailloud P, Beauchamp NJ, et al. Combined stent placement and thrombolysis in acute vertebrobasilar ischemic stroke. Am J Neuroradiol. 2003; 24 (9) 1827-1833.

4 Matsubara N, Miyachi S, Kojima T, et al. Acute vertebral artery origin occlusion leading to basilar artery thrombosis successfully treated by angioplasty with stenting and intracranial fibrinolysis. Neurointervention. 2013; 8 (1): 41-45. doi: 10.5469/neuroint.2013.8.1.41 to vertebral arterial dissection. These results suggest that mechanical thrombectomy in patients with acute stroke resulting from arterial dissection is feasible, safe, and may be associated with favorable functional outcomes.

Stenting of acute vertebral artery origin occlusion and thrombectomy of a BA thrombus were successfully performed. The use of a balloon-assisted guiding catheter to pass the vertebral artery stent was effective. The use of this technique in other related cases could be beneficial for patient prognosis in future cases.

\section{Acknowledgements}

The authors thank Dr Hiroshi Shimoda, MD; Dr Yuya Goto, MD and Dr Akihito Kutsuna, MD, of the Department of Internal Medicine, Divisions of Neurology, Nippon Medical School, Tokyo, Japan, for their technical assistance.

5 Fields JD, Lutsep HL, Rymer MR, et al. Endovascular mechanical thrombectomy for the treatment of acute ischemic stroke due to arterial dissection. Interv Neuroradiol. 2012; 18 (1): 74-79.
Dr Arata Abe, $\mathrm{PhD}$

Department of Internal Medicine

Divisions of Neurology

Nippon Medical School

Tokyo, Japan

Tel.: +81358157964

Fax: +81358157965

E-mail: abe@nms.ac.jp 